\title{
VALIDAÇÃO DA ESCALA DE UTILIZAÇÃO DAS TECNOLOGIAS DIGITAIS NA GESTÃO ESCOLAR
}

\author{
VALIDATION OF THE SCALE OF DIGITAL TECHNOLOGIES USE \\ IN SCHOOL MANAGEMENT
}

\section{VALIDACIÓN DE LA ESCALA DE USO DE TECNOLOGÍAS DIGITALES EN LA GESTIÓN ESCOLAR}

João Piedade ${ }^{1}$, Nuno Dorotea ${ }^{2}$

\section{RESUMO}

O presente artigo relata o processo de validação de uma escala de frequência na utilização das tecnologias digitais pelos diretores de escolas Portuguesas nas suas práticas profissionais. A escala em questão foi desenvolvida pelos autores e previamente validada junto a 3 especialistas em gestão e administração escolar, e posteriormente aplicada à amostra constituída por 133 diretores de escolas do ensino básico e secundário português. A qualidade métrica da escala foi avaliada pela análise de três indicadores: (i) a sensibilidade dos itens mediante o cálculo dos valores da assimetria e da curtose, (ii) a validade recorrendo à análise fatorial exploratória com rotação varimax, e (iii) a consistência interna por meio do cálculo do alpha de Cronbach. Os resultados são reveladores de boa qualidade métrica da escala, o que poderá permitir a sua utilização com um grau de confiança favorável.

PALAVRAS-CHAVE: Escala de utilização das tecnologias digitais na gestão escolar, Diretores escolares, Tecnologias digitais na educação, Gestão escolar, Análise fatorial.

\begin{abstract}
This article reports the validation process of a frequency scale in the use of digital technologies by Portuguese school principals in their professional practices. This scale developed by the authors was previously validated by 3 specialists in school management, and then applied to the sample composed of 133 school principals of Portuguese primary and secondary schools. The metric quality of the scale was evaluated by the analysis of three indicators: (i) the sensitivity of the items by calculating the values of asymmetry and kurtosis, (ii) the validity using exploratory factor analysis with varimax rotation, and (iii) the internal consistency by calculating Cronbach's alpha. The results are revealing good quality metric scale and can allow its use with a favorable degree of confidence.
\end{abstract}

KEYWORDS: Digital technologies use scale in school management, School principals, Digital technologies in education, School management, Factor analysis.

\footnotetext{
${ }^{1}$ Doutor em Educação na especialidade de Tecnologias da Informação e Comunicação na Educação Universidade de Lisboa. Professor Auxiliar convidado do Instituto de Educação da Universidade de Lisboa Alameda da Universidade. Lisboa, Portugal. E-mail: jmpiedade@ie.ulisboa.pt

${ }^{2}$ Doutor em Educação na especialidade de Tecnologias da Informação e Comunicação na Educação Universidade de Lisboa. Professor Auxiliar convidado do Instituto de Educação da Universidade de Lisboa Alameda da Universidade. Lisboa, Portugal. E-mail: nmdorotea@ie.ulisboa.pt

Submetido em: 02/12/2019 - Aceito em: 07/11/2020
}

(C) ETD-Educação Temática Digital Campinas, SP $\quad$ v.23 $\quad$ n.3 $\quad$ p. 757-775 jul./set.2021




\section{RESUMEN}

Este artículo informa sobre el proceso de validación de una escala de frecuencia en el uso de tecnologías digitales por parte de los directores de las escuelas portuguesas en sus prácticas profesionales. La escala en cuestión fue desarrollada por los autores y previamente validado con 3 especialistas en gestión y administración escolar, y posteriormente aplicado a la muestra de 133 directores de escuelas primarias y secundarias portuguesas. La calidad métrica de la escala se evaluó mediante el análisis de tres indicadores: (i) sensibilidad del elemento mediante el cálculo de los valores de asimetría y curtosis, (ii) validez mediante análisis de factores exploratorios con rotación varimax, y (iii) consistencia interna calculando el alfa de Cronbach. Los resultados revelan una buena calidad métrica de la escala, que puede permitir su uso con un grado favorable de confianza.

PALAVRAS-CLAVE: Escala de uso de tecnologías digitales en la gestión escolar, Directores de escuela, Tecnologías digitales en educación, Gestión escolar, Análisis factorial.

\section{INTRODUÇÃO}

A revolução digital apresenta de forma natural grandes desafios à escola e aos agentes educativos, especificamente ao nível da educação dos alunos para a sociedade da informação ou do conhecimento. A escola não pode ser alheia ao desenvolvimento da sociedade e aos desafios que essa evolução acarreta, em especial na figura dos seus órgãos de gestão e diretores escolares. $\mathrm{O}$ cotidiano das escolas permite, por um lado, o acesso quase ilimitado à informação e ao conhecimento; e por outro lado, mostra-se incapaz de garantir a muitos jovens, os instrumentos mínimos no campo da literacia digital. É importante refletir sobre até que ponto as tecnologias podem ser um recurso de combate ao insucesso escolar ou a um desinteresse pela própria escola ou, se pelo contrário, podem ser um instrumento destinado a acentuar as distâncias entre aqueles que mais sabem e os que menos sabem.

Alguns estudos têm evidenciado o papel determinante que os diretores escolares têm no processo de integração das tecnologias nos contextos dessas instituições. As suas crenças, atitudes e visões estratégicas, bem como o papel de liderança tecnológica que podem exercer são apresentados como fatores relevantes e potencializadores da utilização das tecnologias nos espaços escolares. Desse modo, assume-se que no contexto atual, social e tecnologicamente enriquecido, os líderes escolares devem procurar ser catalisadores da mudança e inovação, existindo, no entanto, um longo caminho a percorrer.

Considerando a importância atribuída aos diretores escolares nos processos de modernização tecnológica de suas escolas, é importante conhecer os níveis de utilização das tecnologias digitais nas suas práticas profissionais. Assim, definiu-se como objetivo analisar os índices de utilização das tecnologias apresentados por diretores de escolas públicas portuguesas em várias dimensões das suas estratégias e atividades. 
$\mathrm{Na}$ ausência de um instrumento consolidado e testado em contexto português para medir tais utilizações, desenvolveu-se um instrumento de aferição, organizado em uma escala de frequência, do tipo self-report, considerando 5 dimensões da atividade profissional dos diretores escolares: i) comunicação; ii) planejamento; iii) dinamização de reuniões; iv) avaliação; e v) gestão. A identificação dessas dimensões resulta da análise das competências e responsabilidades definidas pelos normativos legais que regulamentam a organização e a gestão escolar em território português, dentre eles o Decreto-lei N. 9 75/2008 de 22 de abril e N.o 137/2012 de 2 de julho.

Neste artigo relata-se o processo de construção da escala de utilização das tecnologias na gestão escolar e, consequentemente, o processo e validação e avaliação de sua qualidade métrica.

\section{ENQUADRAMENTO TEÓRICO}

\subsection{Utilização das Tecnologias na Gestão Escolar}

No contexto português, a investigação sobre as crenças, atitudes e utilização das tecnologias digitais na gestão e administração escolar ou nas atividades profissionais dos diretores escolares é praticamente inexistente. Esta ausência pode, em parte, ser justificada pela escassez de iniciativas e programas de formação na área das tecnologias digitais dirigidos especificamente aos diretores escolares. De fato, a oferta de formação contínua nessa área tem assumido como público privilegiado os professores.

Em contrapartida, em nível internacional, nos últimos anos alguns autores têm produzido evidências sobre o papel dos diretores escolares na integração educativa das tecnologias, bem como sobre as suas crenças e práticas de utilização em particular (ABDULLAH; DEWITT; ALIAS, 2013; AFSHARI et al., 2010; CAKIR, 2012; ÇAKIR, 2014; KHALID; BUUS, 2014; MAKEWA et al., 2013; MOOLENAAR; SLEEGERS, 2015; SEYAL, 2015; STUART; MILLS; REMUS, 2009; WONG; KHADIJAH, 2017).

Abdullah, DeWitt e Alias (2013) reforçam a importância das crenças pessoais dos diretores escolares como fatores que influenciam na adoção e implementação das tecnologias digitais nas suas escolas. Desse modo, os mesmos autores reportam que os diretores escolares com uma forte visão estratégica sobre o papel das tecnologias digitais na promoção de práticas pedagógicas inovadoras, assumem um papel preponderante na criação de uma cultura de utilização das tecnologias nos seus contextos escolares. O mesmo grau de importância atribuído às crenças e atitudes pessoais é referido por Çakir (2014, p. 736) quando indica que "[...] the success of any attempt to implement technology in educational programs depends strongly upon the support and attitudes of teachers and principals [...]".

(C) ETD-Educação Temática Digital Campinas, SP $\quad$ v.23 n.3 $\quad$ p. 757-775 jul./set.2021 
Afshari et al. (2010) apontam o papel do diretor como fator crítico para a transformação tecnológica do currículo escolar, reforçando a necessidade de reconhecer iniciativas e programas de formação em liderança transformacional desenvolvidas, gestão e tecnologia. Vários outros autores acentuam, igualmente, a necessidade de o diretor assumir uma 'liderança transformacional' capaz de promover o envolvimento e compromisso por parte da sua comunidade escolar na direção a uma efetiva adoção e utilização das tecnologias digitais e, consequente, modernização de práticas (KHALID; BUUS, 2014; SEYAL, 2015; WONG; LI, 2008).

Peled, Kali e Dori (2011), no âmbito de um programa longitudinal de desenvolvimento profissional docente, identificaram que a utilização das tecnologias digitais pelos professores, o suporte e o incentivo por parte dos diretores escolares causam um impacto relevante no contexto escolar. Como base nos resultados do seu estudo, os autores recomendam que os diretores escolares se envolvam nos programas de desenvolvimento profissional dos seus professores e, ainda, que procurem constituir nas suas escolas equipes de docentes com potencial para promover e liderar a integração das tecnologias (PELED; KALI; DORI, 2011). Na mesma linha de argumentação, Afshari et al. (2012) consideram que as competências na utilização das tecnologias pelos diretores escolares são um fator-chave para a criação de uma forte liderança tecnológica nos seus contextos escolares. Assim, os diretores, para além de procurar compreender o papel das tecnologias nas suas práticas profissionais, devem envolver-se em iniciativas de formação que lhes permitam desenvolver competências necessárias para impulsionar o uso das tecnologias digitais nas suas práticas profissionais e nas práticas pedagógicas dos professores (STUART; MILLS; REMUS, 2009).

No início do século, Mulkeen (2001), em um estudo desenvolvido junto a escolas irlandesas concluía que as práticas de utilização das tecnologias por parte dos líderes escolares, no tocante ao nível da comunicação regular por correio eletrônico, tendiam a exercer influência positiva nas práticas de utilização dos demais professores.

Estudos desenvolvidos em contexto internacional têm indicado diferentes potencialidades proporcionadas pelas tecnologias no incremento da qualidade dos processos de gestão escolar (HADDAD; JURICH, 2002; MAKI, 2008; ZAINALLY, 2008; SAITI; PROKOPIADOU, 2009). Nesse domínio, Haddad e Jurich (2002) apresentam 3 ideias chave: a) permitem uma gestão mais eficiente de processos ligados à gestão, à supervisão do progresso dos alunos e à gestão de recursos; b) tornam os processos de transações administrativos mais fáceis e 'amigáveis'; e c) promovem a comunicação entre a gestão, os professores, os alunos, os pais e toda a comunidade. Zainally (2008, p. 283) indica que "[...] ICT provides several facilities for educational administrators to perform their tasks [...]". No mesmo sentido, Maki (2008 apud MAKEWA et al., 2013, p. 48) afirma que "[...] ICT plays a vital role in supporting

$\begin{array}{llllll}\text { (C) ETD-Educação Temática Digital } & \text { Campinas, SP } & \text { v.23 } & \text { n.3 } & \text { p.757-775 } & \text { jul./set.2021 }\end{array}$ 
powerful, eficient management and administration in education sector [...]".

Saiti e Prokopiadou (2009), em um estudo sobre o impacto das tecnologias digitais na administração escolar realizado com escolas gregas, reportam que a utilização das tecnologias no contexto escolar pode ser sinônimo de modernização e inovação nas práticas letivas, mas também nas atividades de administração escolar. Mencionando, ainda, que as tecnologias digitais proporcionam um conjunto de ferramentas e de ' $e$-services' que facilitam transações administrativas e tornam mais eficientes os processos de partilha de informação entre a comunidade educativa.

A percepção de eficácia e a utilização das tecnologias digitais por parte dos diretores escolares foi igualmente analisada por Arokiasamy, Abdullah e Ismail (2015). O estudo, de caráter descritivo e exploratório, desenvolvido junto a 520 diretores de escolas secundárias da Malásia procurou correlacionar as perceções do estilo de liderança e a utilização das tecnologias pelos diretores escolares. $O$ processo de coleta de dados foi organizado com base em um questionário constituído por duas partes: a) escala de autoperceção sobre o nível de utilização das tecnologias digitais; e b) características pessoais dos diretores escolares, competência percebida na utilização do computador e estilo de liderança (transformacional e transacional). Os resultados revelaram índices moderados de utilização das tecnologias digitais nas várias dimensões analisadas e níveis elevados na perceção cultural sobre a relevância dos computadores no contexto educativo. Os autores concluíram, ainda, que apesar dos resultados positivos, os diretores escolares necessitam de desenvolver competências sobre a utilização de bases de dados, folhas de cálculo, aplicações multimédia, redes e ferramentas de comunicação de modo a incrementar os níveis de utilização nas suas práticas profissionais. No mesmo país, o estudo realizado por Hoque, Razak e Zohora (2012) procurou conhecer as práticas de utilização das tecnologias da informação e comunicação por professores e diretores de escolas. A investigação envolveu a participação de 215 professores e 45 diretores escolares e teve como propósito obter dados sobre três aspectos: a) políticas de incentivo à implementação das tecnologias; b) disponibilidade e acessibilidade a recursos TIC; e c) eficiência e capacidade de utilização das tecnologias. A análise dos dados revelou que a utilização das tecnologias na gestão escolar das escolas analisadas, é ainda muito limitada e, de um modo geral, as tecnologias não são usadas de forma efetiva e eficiente.

Çakir (2014), em um estudo desenvolvido junto a 460 professores e 308 diretores do ensino pré-escolar da Turquia, procurou avaliar o interesse, a ansiedade e a utilização das tecnologias nas suas práticas profissionais. O autor concluiu que professores e diretores apresentam níveis elevados na dimensão de utilização das tecnologias e níveis mais baixos na variável ansiedade. Ressaltou, ainda, que esses resultados se justificam pelo fato de que quanto mais elevados forem os índices de utilização das tecnologias, menor será o índice de

$\begin{array}{llllll}\text { (C) ETD-Educação Temática Digital } & \text { Campinas, SP } & \text { v.23 } & \text { n.3 } & \text { p.757-775 } & \text { jul./set.2021 }\end{array}$ 
ansiedade perante as mesmas, salientando, assim, a relação entre estes dois construtos.

Em 2015, Gastelú, Kiss e Dominguez (2015) desenvolveram um estudo, no qual participaram cerca de $20 \%$ dos diretores escolares de escolas básicas de Veracruz no México, em que buscaram analisar as atitudes dos diretores perante as tecnologias digitais. Relativamente à utilização dos computadores pela comunidade escolar, $74 \%$ dos participantes afirmou que estes equipamentos são usados pelos diretores escolares, $60 \%$ disse que são usados pelos professores, $37 \%$ reportou que são usados pelos estudantes e $9 \%$ que são usados pelo restante staff da escola. Os autores concluíram que os diretores escolares apresentam uma atitude relativamente positiva às tecnologias e que conseguem as utilizar, sobretudo na gestão de tarefas diárias. No entanto, salientam "[...] integration process of ICT in public primary schools in Veracruz city is a pending task to be resolved [...]" (GASTELÚ; KISS; DOMINGUEZ, 2015, p. 822), sendo necessário providenciar infraestruturas tecnológicas, desenvolver planos de formação para diretores e professores, bem como desenhar políticas educativas nacionais que promovam a presença das tecnologias nas atividades das escolas.

Em síntese, pela revisão de literatura fica evidente que os diretores escolares têm um papel determinante no processo de integração das tecnologias nos seus contextos escolares. As suas crenças, atitudes e visão estratégica, bem como o papel de liderança tecnológica que podem exercer, são apresentados como fatores relevantes e potenciadores da utilização das tecnologias nas suas escolas. Assim, considera-se, que no contexto atual, social e tecnologicamente enriquecido, os líderes escolares devem procurar ser catalisadores de mudança e inovação nos seus contextos escolares, existindo, no entanto, um longo caminho a percorrer e um conjunto de fatores que importa garantir. Relativamente à utilização das tecnologias digitais nas práticas de gestão e administração escolar, e apesar de os diretores escolares apresentarem crenças e atitudes positivas em relação às tecnologias, o seu uso resume-se à utilização de aplicações de processamento de texto, apresentações eletrônicas, correio eletrônico, internet e em alguns casos folha de cálculo. A utilização de outros tipos de aplicações da web 2.0 ou ferramentas específicas para a gestão tende a não ser reportada. Os vários estudos analisados apontam, ainda, a necessidade de serem desenvolvidos, para além de incentivos e políticas para a utilização das tecnologias, programas de desenvolvimento de competências em tecnologias e inovação especialmente dirigidos a diretores escolares.

\subsection{Escala de Utilização das Tecnologias Digitais na Gestão Escolar}

A escala de utilização das tecnologias digitais na gestão escolar, desenvolvida no âmbito de um programa de doutoramento em educação, apresenta-se como um instrumento multidimensional, com o qual se pretende analisar a frequência de utilização das tecnologias

(C) ETD-Educação Temática Digital Campinas, SP $\quad$ v.23 $\quad$ n.3 $\quad$ p. 757-775 jul./set.2021 
pelos diretores escolares nas várias dimensões da sua atividade profissional. Acredita-se, deste modo, que os diretores escolares possam utilizar as tecnologias digitais com diferente

intensidade, considerando as diferentes especificidades das suas práticas profissionais.

Embora conscientes que as competências e dimensões do trabalho do diretor escolar podem não se esgotar nas categorias definidas nos normativos legais, para a elaboração da escala analisaram-se as competências e perfil profissional dos diretores escolares definidas nos Decretos-lei N.ㅇ 75/2008 de 22 de abril e N.ㅇ 137/2012 de 2 de julho. Estes normativos legais estabelecem e regulamentam o regime de autonomia, administração e gestão dos estabelecimentos públicos da educação pré-escolar e dos ensinos básico e secundário, incluindo a definição das competências e responsabilidades do diretor escolar. Ao diretor escolar é atribuída a gestão financeira, administrativa e pedagógica da escola ou agrupamento de escolas. O decreto-lei n.o 75/2008 de 22 de abril veio reforçar claramente as competências do diretor escolar, enquanto entidade com plena responsabilidade pela gestão e administração da escola. Nessa linha de reforço das responsabilidades do diretor escolar, Supico (2013) acentua, com base numa análise comparativa dos normativos legais, que as competências e responsabilidades do diretor escolar têm vindo a ser progressivamente reforçadas desde 1991. Assim, o diretor enquanto entidade máxima escolar assume um papel de relevo em todas as áreas de administração, gestão e pedagógica, incluindo as relacionadas com a integração educativa das tecnologias nos seus contextos educativos.

Considerando o exposto, propôs-se um instrumento organizado em uma escala de frequência, composto por 22 itens estruturado em 5 dimensões:

1. Comunicação: Utilização das tecnologias pelos diretores para comunicação com os vários agentes educativos e com a comunidade. Pretende-se, nesta dimensão, medir o grau de utilização institucional das tecnologias para comunicação com organismos centrais e regionais (ex. Ministério da Educação, Direções-gerais, Direções Regionais de Educação, Direções Gerais, etc.), com o corpo docente das suas escolas, com os seus alunos e com a comunidade envolvida na escola.

2. Planejamento: Utilização das tecnologias para planejamento da atividade profissional. Nesta dimensão, procura-se medir o grau de utilização das tecnologias para atividades de planejamento da atividade profissional dos diretores escolares, como a organização de atividades, reuniões de trabalho e gestão de agenda. 
3. Dinamização de reuniões: Utilização das tecnologias para dinamização e condução de reuniões de trabalho. Nesta dimensão, pretende-se medir o grau de utilização das tecnologias no suporte à promoção e reuniões de trabalho colaborativas, na realização de reuniões de trabalho a distância, na execução de apresentações eletrônicas em reuniões e na utilização de aplicações on-line para gestão documental.

4. Avaliação: Utilização das tecnologias para avaliação de docentes e não docentes, autoavaliação de escola. Análise do grau de utilização das tecnologias na realização de investigações junto à comunidade escolar, na promoção de mecanismos e práticas de autoavaliação de escola, na avaliação dos docentes e dos funcionários não docentes e no monitoramento dos indicadores de qualidade escolar.

5. Gestão: utilização das tecnologias para realização de atividades de gestão. Pretende-se, nesta dimensão, medir o grau de utilização das tecnologias em atividades de gestão escolar como a elaboração e administração do orçamento, a seleção e recrutamento de pessoal docente e não docente, a constituição de turmas, a distribuição de serviço, entre outras.

A distribuição dos itens por cada uma das dimensões encontra-se representada na tabela 1, cumprindo o que reforça Maroco (2014), quando indica que cada dimensão deve conter um mínimo de 3 itens.

TABELA 1 - Organização dos itens da escala por dimensão

\begin{tabular}{ccccc}
\hline $\begin{array}{c}\text { Dimensão 1 } \\
\text { Comunicação }\end{array}$ & $\begin{array}{c}\text { Dimensão 2 } \\
\text { Planificação }\end{array}$ & $\begin{array}{c}\text { Dimensão 3 } \\
\text { Reuniões }\end{array}$ & $\begin{array}{c}\text { Dimensão 4 } \\
\text { Avaliação }\end{array}$ & $\begin{array}{c}\text { Dimensão 5 } \\
\text { Gestão }\end{array}$ \\
\hline$P 1, P 2, P 3, P 4, P 5$, & $P 7, P 8, P 13$ & $P 9, P 11, P 14$, & $P 12, P 15$, & $P 17, P 19$, \\
$P 6, P 10$ & & $P 22$ & $P 16, P 18$ & $P 20, P 21$ \\
\hline
\end{tabular}

Os itens da escala são constituídos por 5 opções de resposta de formato likert, variando entre "Muito Raramente" e "Muito Frequentemente", cotadas entre 1 e 5 respetivamente. Os valores mais altos revelam índices mais elevados de utilização das tecnologias digitais e os valores mais baixos indicam índices de utilização mais reduzidos. 


\section{MÉTODO}

\subsection{Participantes}

A amostra não probabilística foi organizada com diretores de escolas e agrupamentos escolares de Portugal continental em exercício de funções. Para constituição da amostra uma versão da escala disponível on-line foi enviada, por meio dos e-mails institucionais, aos diretores escolares das 831 escolas e agrupamentos escolares existentes em Portugal. 0 número de respostas válidas foi de 133 , o que corresponde a cerca de $16 \%$ do universo total de diretores escolares.

A amostra é composta por $52.63 \%$ de diretores do gênero masculino e $47.37 \%$ do gênero feminino, $51.13 \%$ tem entre 40 e 60 anos, $80 \%$ exerce o cargo há mais de 5 anos e $75 \%$ apresenta formação especializada em gestão e administração escolar. Na amostra constituída existe representatividade de diretores escolares de todas as regiões e distritos de Portugal continental.

\subsection{Procedimentos}

Após o processo de desenvolvimento da escala de utilização das tecnologias na gestão escolar, procedeu-se à sua validação por especialistas e à sua pré-testagem junto de um grupo de diretores escolares. Mediante os contributos de três acadêmicos especialistas na área da gestão e administração escolar, partiu-se à reformulação de 2 itens da escala e à eliminação de um outro, originado assim a segunda versão do instrumento. Os três especialistas consideraram adequadas as 5 dimensões definidas. Em um segundo momento, procedeu-se à validação da segunda versão da escala junto de 10 diretores escolares experientes no desempenho do cargo. Os testes junto desse grupo basearam-se na análise do critério de fidelidade que revelou um elevado índice de consistência interna ( $\alpha=.97)$. A análise da qualidade métrica da escala e os comentários e sugestões dos diretores levaram à reformulação dos itens $1,13,17$ e 21 , originando desta forma a versão final do instrumento.

A versão final da escala constituída por 22 itens (Tabela 2), organizados em 5 dimensões foi aplicada à amostra constituída pelos 133 diretores escolares. Seguidamente à coleta de dados, foi utilizada a aplicação SPSS (versão 25) para realização de todos os procedimentos estatísticos com vistas à validação da escala. 


\section{RESULTADOS}

Inerente ao processo de construção da escala de utilização das tecnologias digitais nas práticas de gestão escolar, revelou-se necessário proceder à análise da sensibilidade, validade e confiabilidade da mesma, procurando, assim, eliminar quaisquer itens que se apresentem menos discriminativos. A qualidade métrica dos instrumentos foi delineada considerando os seguintes critérios (ALMEIDA; FREIRE, 2017; MAROCO, 2014): a) sensibilidade, procurando avaliar a capacidade do instrumento para detectar as variações nas respostas dos sujeitos inquiridos. Analisa, assim, se os dados obtidos através das respostas dos sujeitos diferenciam as posições assumidas pelos mesmos (aferido com base em testes de Assimetria e Achatamento); b) validade, avaliando o quão válidos de analisar se revelam os dados recolhidos, procurando perceber se o instrumento mede aquilo que pretende medir e se os dados recolhidos refletem a realidade (testado com base em procedimentos de análise fatorial); e c) fidelidade: procurando avaliar a consistência e exatidão do processo de medida, analisando, por exemplo, se diferentes investigadores, nas mesmas circunstâncias ou a aplicação do instrumento aos mesmos sujeitos, em momentos diferentes, revelariam os mesmos resultados (testado com base no Coeficiente alpha de Cronbach). Apresentam-se, nos pontos seguintes os resultados encontrados na sequência do processo de validação dos instrumentos mediante os testes estatísticos anteriormente identificados.

\subsection{Sensibilidade dos Itens, Validade Fatorial e Consistência Interna}

\subsubsection{Sensibilidade}

A análise da sensibilidade dos itens permite verificar se a escala é sensível às variações nas respostas dos participantes. Assim, pretende-se analisar se os itens da escala permitem discriminar os sujeitos com padrões diferentes, com base nas frequências de utilização das tecnologias digitais. A análise realiza-se por meio do cálculo de 2 indicadores, a assimetria e o achatamento (curtose) que apresentam valores aceitáveis entre -3 e +3 e -7 e +7 respetivamente (MAROCO, 2014). Considerando os valores representados na tabela 1, a maioria dos itens que compõem a escala apresentam valores de assimetria e de achatamento aceitáveis, exceto os itens P1, P10 e P17. Apesar de ser aconselhável eliminar esses três itens, decidimos pela sua manutenção dada aos valores de consistência interna da escala à frente referidos. 
TABELA 2 - Valores de assimetria e curtose da escala utilização das tecnologias digitais na gestão escolar $(n=133)$

\begin{tabular}{|c|c|c|}
\hline Variável & Assimetria & Curtose \\
\hline $\begin{array}{l}\text { P1 - Com que frequência utiliza as TIC para comunicar institucionalmente } \\
\text { com organismos centrais e regionais (ex. Ministério da Educação e Ciência, } \\
\text { Direções Regionais de Educação, Direções Gerais, etc.)? }\end{array}$ & -3.30 & 11.72 \\
\hline P2 - Com que frequência utiliza as TIC para comunicar com os docentes? & -2.20 & 4.75 \\
\hline $\begin{array}{l}\text { P3 - Com que frequência utiliza as TIC para comunicar com funcionários não } \\
\text { docentes? }\end{array}$ & -.14 & -1.11 \\
\hline P4 - Com que frequência utiliza as TIC para comunicar com os alunos & -.21 & -.81 \\
\hline $\begin{array}{l}\text { P5 - Com que frequência utiliza as TIC para comunicar com os encarregados } \\
\text { de educação }\end{array}$ & -.06 & -.86 \\
\hline $\begin{array}{l}\text { P6 - Com que frequência utiliza as TIC para comunicar institucionalmente } \\
\text { com organismos Locais (ex. Municípios, Juntas de Freguesias, Associações, } \\
\text { Empresas Locais, etc.)? }\end{array}$ & -2.09 & 5.15 \\
\hline P7 - Com que frequência utiliza as TIC para preparar as reuniões de trabalho? & -2.30 & 6.36 \\
\hline P8 - Com que frequência utiliza as TIC para planificar as atividades & -1.96 & 4.58 \\
\hline $\begin{array}{l}\text { P9 - Com que frequência utiliza as TIC para promover a colaboração nas } \\
\text { reuniões dos vários órgãos da escola/ agrupamento? }\end{array}$ & -1.98 & 4.19 \\
\hline $\begin{array}{l}\text { P10 - Com que frequência utiliza as TIC para divulgar informação relacionada } \\
\text { com a gestão? }\end{array}$ & -2.52 & 7.55 \\
\hline $\begin{array}{l}\text { P11 - Com que frequência utiliza as TIC como suporte a reuniões de trabalho } \\
\text { a distância? }\end{array}$ & -.61 & -.76 \\
\hline $\begin{array}{l}\text { P12 - Com que frequência utiliza as TIC para realizar inquéritos à comunidade } \\
\text { escolar }\end{array}$ & -.60 & -.30 \\
\hline P13 - Com que frequência utiliza as TIC para gestão de agenda ou trabalho? & -.58 & -.38 \\
\hline $\begin{array}{l}\text { P14 - Com que frequência utiliza as TIC para realização de apresentações } \\
\text { eletrónicas nas reuniões dos órgãos da escola/agrupamento }\end{array}$ & -1.81 & 3.24 \\
\hline $\begin{array}{l}\text { P15 - Com que frequência utiliza as TIC para a análise estatística dos } \\
\text { resultados escolares? }\end{array}$ & -2.29 & 5.46 \\
\hline $\begin{array}{l}\text { P16 - Com que frequência utiliza as TIC para calcular e registar a avaliação } \\
\text { dos funcionários docentes e não docentes? }\end{array}$ & -1.69 & 2.55 \\
\hline $\begin{array}{l}\text { P17 - Com que frequência utiliza plataformas e aplicações online para envio } \\
\text { de dados solicitados pela a Tutela (Ex. Estatísticas de no Alunos, Professores, } \\
\text { etc...)? }\end{array}$ & -3.24 & 10.78 \\
\hline
\end{tabular}


DOI: $10.20396 /$ etd.v23i3.8657850

\begin{tabular}{lcc}
\hline Variável & Assimetria & Curtose \\
\hline $\begin{array}{l}\text { P18 - Com que frequência utiliza as TIC para seleção e recrutamento de } \\
\text { pessoal docente e não docente? }\end{array}$ & -2.27 & 4.98 \\
\hline P19 - Com que frequência utiliza as TIC para a constituição de turmas? & -1.69 & 2.32 \\
\hline $\begin{array}{l}\text { P20 - Com que frequência utiliza as TIC para a distribuição de serviço letivo? } \\
\text { P21 - Com que frequência utiliza as TIC para a elaboração e gestão do } \\
\text { orçamento escolar? }\end{array}$ & -2.24 & 3.54 \\
\hline $\begin{array}{l}\text { P22 - Com que frequência utiliza aplicações online para partilha de } \\
\text { documentos de trabalho com a sua equipa? }\end{array}$ & -1.81 & -1.32 \\
\hline
\end{tabular}

\subsubsection{Análise Fatorial}

No processo de desenvolvimento e validação dos instrumentos de coleta de dados, a validade é um dos principais indicadores da qualidade métrica de um determinado instrumento de medida. Este indicador avalia o quão válidos de analisar se revelam os dados recolhidos, procurando perceber se os itens medem aquilo que pretende medir e se os dados recolhidos refletem a realidade. Neste caso, centramos a análise apenas na validade de construto por meio de processos de análise fatorial exploratória (AFA). A análise fatorial " [...] é uma técnica de análise exploratória de dados que tem por objetivo descobrir e analisar a estrutura de um conjunto de variáveis interrelacionadas de modo a construir uma escala de medida para fatores que de alguma forma controlam as variáveis originais [...]" (MAROCO, 2010 , p. 361). A AFE tem sido um dos procedimentos estatísticos mais utilizados no desenvolvimento e validação de instrumentos psicológicos (FLOYD; WIDAMAN, 1995) e consiste em um conjunto de técnicas multivariadas que têm como objetivo encontrar a estrutura subjacente em uma matriz de dados, bem como determinar o número e a natureza das variáveis latentes (fatores) que melhor representam um conjunto de variáveis observadas (BROWN, 2006).

Para implementação do procedimento de AFE consideramos as cinco dimensões (fatores) definidas para a escala de utilização das tecnologias digitais nas práticas de gestão escolar, com a distribuição dos itens por dimensão representadas na tabela 1.

$\begin{array}{llllll}\text { (C) ETD-Educação Temática Digital } & \text { Campinas, SP } & \text { v.23 } & \text { n.3 } & \text { p.757-775 } & \text { jul./set.2021 }\end{array}$ 
Previamente à análise fatorial exploratória é necessário garantir dois pressupostos, os valores do teste kaiser-Meyer-Olkin Measure of Sampling Adequacy (KMO) superiores a 0.7 e os valores do teste de esferecidadade de Barlet inferiores a .001. A aplicação do teste KMO revelou um valor de .913, o que permite constatar que a recomendação de aplicação do modelo de análise fatorial se revela muito boa (PESTANA; GAGEIRO, 2008). Nesse sentido, a aplicação do teste de esfericidade de Bartlet constatou que as variáveis se apresentam correlacionadas de forma significativa ( $\chi 2=2026.719 ; p=.000$ ). Desse modo, cumprem-se os dois pressupostos de aplicação do modelo de análise fatorial.

Após o cumprimento dos pressupostos anteriores, aplicamos o modelo de AFE, selecionando o método de componentes principais, Rotação Varimax, definindo à partida cinco fatores fixos, que correspondem às 5 dimensões definidas na construção do instrumento. O método de rotação Varimax tem como objetivo obter uma estrutura fatorial, na qual uma e apenas uma das variáveis originais esteja fortemente associada com um único fator e pouco associada com os restantes.

TABELA 3 - Teste de KMO e Bartlett's dos 22 itens da escala

\begin{tabular}{lll}
\hline \multicolumn{3}{c}{ KMO and Bartlett's Test } \\
\hline $\begin{array}{l}\text { Kaiser-Meyer-Olkin Measure of Sampling } \\
\text { Adequacy }\end{array}$ & .913 \\
\hline & $\begin{array}{l}\text { Approx. Chi- } \\
\text { Square }\end{array}$ & 2026.72 \\
\cline { 2 - 3 } Bartlett's Test of Sphericity & df & 231 \\
\cline { 2 - 3 } & Sig. & .000 \\
\hline
\end{tabular}

A análise fatorial permitiu verificar que os 5 fatores, definidos anteriormente, explicavam cerca de $73 \%$ da variabilidade total da escala de utilização das tecnologias na gestão escolar, o que poderá ser considerado aceitável e favorável (MAROCO, 2014). 
TABELA 4 - Análise fatorial com rotação varimax

\begin{tabular}{|c|c|c|c|c|c|c|}
\hline Item & 1 & 2 & 3 & 4 & 5 & Comunalidades \\
\hline$P 1$ & .778 & & & & & .793 \\
\hline$\overline{P 2}$ & .774 & & & & & .733 \\
\hline$\overline{P 3}$ & .558 & & & & & .546 \\
\hline$P 4$ & .434 & & & & & .818 \\
\hline$P 5$ & .528 & & & & & .809 \\
\hline$\overline{P 6}$ & .621 & & & & & .657 \\
\hline$P 7$ & & .728 & & & & .745 \\
\hline P8 & & .469 & & & & .789 \\
\hline$\overline{P 9}$ & & & .756 & & & .728 \\
\hline$P 10$ & .786 & & & & & .713 \\
\hline$P 11$ & & & .428 & & & .723 \\
\hline$P 12$ & & & & .587 & & .717 \\
\hline$P 13$ & & .641 & & & & .657 \\
\hline$P 14$ & & & .738 & & & .648 \\
\hline$P 15$ & & & & .810 & & .688 \\
\hline$P 16$ & & & & .814 & & .776 \\
\hline$P 17$ & & & & & .816 & .866 \\
\hline$P 18$ & & & & .759 & & .738 \\
\hline$P 19$ & & & & & .681 & .666 \\
\hline$P 20$ & & & & & .776 & .721 \\
\hline$P 21$ & & & & & .752 & .739 \\
\hline$P 22$ & & & .546 & & & .699 \\
\hline \%Variância explicada & 46.734 & 9.982 & 6.832 & 4.912 & 4.139 & \\
\hline
\end{tabular}

\subsubsection{Consistência Interna}

A confiabilidade de um instrumento de coleta de dados diz respeito à capacidade deste ser consistente, ou seja, a obtenção dos mesmos resultados quando aplicado a públicos com iguais características (MAROCO; GARCIA-MARQUES, 2006). Para avaliar a confiabilidade da escala ou a sua consistência interna selecionou-se o indicador alfa de Cronbach. Os valores de alfa variam entre 0 e 1, e quanto mais próximo de 1 maior será o grau de confiabilidade. Vários autores têm referido esse indicador para a avaliação da consistência interna de instrumentos de medida e têm reportado valores superiores a 0,7 como favoráveis ou recomendáveis (HILL; HILL, 2005; MAROCO, 2014; MAROCO; GARCIA-MARQUES, 2006; PESTANA; GAGEIRO, 2008).

$\begin{array}{llllll}\text { (C) ETD-Educação Temática Digital } & \text { Campinas, SP } & \text { v.23 } & \text { n.3 } & \text { p.757-775 } & \text { jul./set.2021 }\end{array}$ 
Considerando a multidimensionalidade da escala, procedemos à análise da consistência interna para a globalidade da escala (22 itens), representada na tabela 5 e para cada uma das 5 dimensões (fatores), representada na tabela 6. A análise da consistência interna da escala revelou um coeficiente alpha de Cronbach total de .935, mostrando, assim elevada consistência interna. Analisados os coeficientes para cada um dos itens da escala,

considerou-se manter os 22 itens pois a confiabilidade da escala não melhoraria com a eliminação de qualquer um dos itens. Olhando individualmente cada um dos fatores verificam-se, igualmente, índices de consistência interna favoráveis.

TABELA 5 - Resultados obtidos do Coeficiente de Fiabilidade pelo alpha de Cronbach para globalidade da escala

\begin{tabular}{lll}
\hline & Reliability Statistics & \\
\hline Alpha Cronbach & & $n$ \\
\hline & .935 & 22 \\
\hline
\end{tabular}

TABELA 6 - Resultados obtidos do Coeficiente de fiabilidade pelo alpha de Cronbach para cada um dos 5 fatores definidos

\begin{tabular}{lccccc}
\hline & $\begin{array}{c}\text { Dimensão 1 } \\
\text { Comunicação }\end{array}$ & $\begin{array}{c}\text { Dimensão 2 } \\
\text { Planificação }\end{array}$ & $\begin{array}{c}\text { Dimensão 3 } \\
\text { Reuniões }\end{array}$ & $\begin{array}{c}\text { Dimensão 4 } \\
\text { Avaliação }\end{array}$ & $\begin{array}{c}\text { Dimensão 5 } \\
\text { Gestão }\end{array}$ \\
\hline Itens & $\begin{array}{c}P 1, P 2, P 3, \\
P 4, P 5, P 6, \\
P 10\end{array}$ & $P 7, P 8, P 13$ & $\begin{array}{c}P 9, P 11, \\
P 14, P 22\end{array}$ & $\begin{array}{c}P 12, P 15, \\
P 16, P 18\end{array}$ & $\begin{array}{c}P 17, P 19, \\
P 20, P 21\end{array}$ \\
\hline $\begin{array}{l}\text { Alpha } \\
\text { Cronbach }\end{array}$ & .784 & .725 & .753 & .816 & .890 \\
\hline
\end{tabular}




\section{CONCLUSÕES}

No presente artigo apresentaram-se os procedimentos de validação de uma Escala que pretende medir a frequência de utilização das tecnologias digitais por Diretores Escolares Portugueses em suas práticas profissionais.

Os resultados encontrados permitem constatar que a Escala desenvolvida, validada por especialistas e por uma amostra constituída por 133 diretores escolares, apresenta bons índices de sensibilidade dos itens e uma estrutura fatorial consistente para os cinco fatores (dimensões) definidas. A análise da confiabilidade de cada dimensão e da escala no seu conjunto revelou elevada consistência interna.

A qualidade métrica da escala permite a sua utilização com confiança para medir os índices de frequência de utilização das tecnologias digitais pelos diretores escolares nas várias dimensões das suas atividades profissionais e de gestão. Apesar da boa qualidade métrica revelada no processo de validação da escala, considera-se necessário levar a cabo novos estudos, junto de novas amostras, que permitam atualizar, aprimorar e validar a escala de utilização das tecnologias nas práticas de gestão escolar. Os resultados obtidos em novos processos de validação irão permitir estabelecer comparações com os resultados e as conclusões apresentadas neste estudo, corroborando-os (ou contradizendo-os), ao mesmo tempo que permitirão construir e colocar disponível um instrumento que efetivamente se encontra em falta. Uma das constatações que verificamos, aquando da construção da escala, foi a efetiva ausência de instrumentação disponível para analisar as práticas de utilização das tecnologias nas práticas das direções escolares. Com estes novos estudos de validação, poderá ser disponibilizado um instrumento mais credível, fiável que permita um maior grau de generalização dos resultados. 


\section{REFERÊNCIAS}

ABDULLAH, Nur Ain Wong; DEWITT, Dorothy; ALIAS, Norlidah. School Improvement Efforts and Challenges: a case study of a principal utilizing information communication technology. Procedia - Social and Behavioral Sciences, v. 103, p. 791-800, 2013.

AFSHARI, Mojgan et al. Computer use by school principals. Turkish Online Journal of Educational Technology, v. 9, n. 3, p. 8-25, 2010.

AFSHARI, Mojgan et al. Transformational leadership role of principals in implementing informational and communication technologies in schools. Life Science Journal, v. 9, n. 1, p. 281-284, 2012.

ALMEIDA, Leandro; FREIRE, Teresa. Metodologia de investigação em Psicologia e Educação. Braga: Psiquilíbrios, 2017.

AROKIASAMY, Anantha Raj; ABDULLAH, Abdul Ghani Kanesan bin; ISMAIL, Aziah. Correlation between cultural perceptions, leadership style and ICT usage by school principals in Malaysia. Procedia - Social and Behavioral Sciences, v. 176, p. 319-332, 2015.

BROWN, Timothy. Confirmatory factor analysis for applied research. New York: The Guilford Press, 2006.

CAKIR, Recep. Technology integration and technology leadership in schools as learning organizations. The Turkish Online Journal of Educational Technology, v. 11, n. 4, p. 273282, 2012.

ÇAKIR, Turan. The attitudes of preschool teachers and principals towards computer using. Anthropologist, v. 18, n. 3, p. 735-744, 2014.

FLOYD, Frank; WIDAMAN, Keith. Factor analysis in the development and refinement of clinical assessment instruments. Psychological Assessment, v. 7, n. 3, p. 286-299, 1995.

GASTELÚ, Carlos Arturo; KISS, Gábor; DOMíNGUEZ, Agustín Lagunes. Level of competencies at the university. Procedia - Social and Behavioral Sciences, v. 174, p. 137-142, 2015.

HADDAD Wadi; JURICH Sonia ICT for education: potential and potency: technologies for education: potentials, parameters and prospects. Washington DC: Academy for Educational Development, 2002.

HILL, Manuela Magalhães; HILL, Andrew. Investigação por questionário. Lisboa: Edições Sílabo, 2008.

HOQUE, Kazi Enamul; RAZAK, Ahmad Zabidi Abul; ZOHORA, Mosa Fatema. ICT Utlilization among school teachers and principals in Malaysia. International Journal of Academic Research in Progressive Education and Development, v. 1, n. 4, p. 17-34, 2012. 
KHALID, Saifuddin; BUUS, Lillian. A theoretical framework mapping barriers of integration and adopting educational technology. Research and Practice in Technology Enhanced Learning, p. 1-19, 2014.

MAKEWA, Lazarus; MEREMO, Jackson; ROLE, Elizabeth; ROLE, Jesse. ICT in secondary school administration in rural Southern Kenya: An Educator's eye on its importance and use. International Journal of Education and Development Using Information and Communication Technology, v. 9, n. 2, p. 48-63, 2013.

MAKI, Christiana. Information and communication technology for administration and management for secondary schools in Cyprus. Journal of Online Learning and Teaching, $v$. 4, n. 3, p. 18-20, 2008.

MAROCO, João. Análise estatística com o SPSS Statistics. Pêro Pinheiro: Gráfica Manuel Barbosa \& Filho, 2014.

MAROCO, João. Análise estatística com utilização do SPSS. Lisboa: Edições Sílabo, 2010.

MAROCO, João; GARCIA-MARQUES, Teresa. Qual a fiabilidade do alfa de Cronbach? Questões antigas e soluções modernas? Laboratório de Psicologia, v. 4, n. 1, p. 65-90, 2006.

MOOLENAAR, Nienke; SLEEGERS, Peter. The networked principal: Examining principals' social relationships and transformational leadership in school and district networks. Journal of Educational Administration, v. 53, n. 1, p. 8-39, 2015.

MULKEEN, Aidan. The impact of teacher skills on the integration of ICT in Irish Schools. In: International Conference on Technology and Education. Tallahassee, 2001. Disponível em: http://www.icte.org/To1 Library/T01 170.PDF. Acesso em: 15 nov. 2019.

PELED, Yehuda; KALI Yael; DORI, Yedhudit Judy. School principals' influence on science teachers' tecnhnology implementation: a retrospective analysis. Journal of Leadership in Education, v. 14, n. 2, p. 229-245, 2011.

PESTANA, Maria Helena; GAGEIRO, João Nunes. Análise de dados para ciências sociais: a complementaridade do SPSS. Lisboa: Edições Sílabo, 2008.

SAITI, Anna; PROKOPIADOU, Georgia. Impact of information and communication technologies on school administration: research on the greek schools of secondary education. Lecture Notes in Computer Science, n. 5693, p. 305-316, 2009.

SEYAL, Afzaal. Examining the role of transformational leadership in technology adoption: evidence from bruneian technical \& vocational establishments (TVE). Journal of Education and Practice, v. 6, n. 8, p. 32-43, 2015.

STUART, Lindsay; MILLS, Annette; REMUS, Ulrich. School leaders, ICT competence and championing innovations. Computers \& Education, n. 52, p. 733-741, 2009. 
SUPICO, Maria Luisa. 0 trabalho do diretor nos agrupamentos de escolas: percepções de um gestor. 2013. Dissertação (Mestrado em Ciências da Educação na especialidade de Administração Educacional) - Instituto de Educação da Universidade de Lisboa, Lisboa, 2013.

WONG, Ai Yieng; KHADIJAH, Binto Daud. Technology leadership in malaysia's high performance school. Journal of Education and e-Learning Research, v. 4, n. 1, p. 8-14, 2017.

WONG, Emily M L; LI, S C. Framing ICT implementation in a context of educational change: A multilevel analysis. School Effectiveness and School Improvement, v. 6, n. 1, p. 99-120, 2008.

ZAINALLY, Hossein. Administration of faculties by information and communication technology and its obstacles. International Journal of Education and Information Technologies, v. 2, n. 1, p. 36-42, 2008.

\section{Legislação Consultada}

PORTUGAL. Decreto-lei n. $\mathbf{0 7 5 / 2 0 1 8}$, de $\mathbf{2 2}$ de abril. Aprova o regime de autonomia, administração e gestão dos estabelecimentos públicos da educação pré-escolar e dos ensinos básico e secundário. Lisboa, 2018. Disponível em:

https://dre.pt/application/conteudo/116640899. Acesso em: 16 nov. 2019.

PORTUGAL. Decreto-Lei n. 137/2012, de 02 de julho. Procede à segunda alteração do Decreto-Lei n.o 75/2008, de 22 de abril, que aprova o regime jurídico de autonomia, administração e gestão dos estabelecimentos públicos da educação pré-escolar e dos ensinos básico e secundário. Lisboa, 2018. Disponível em:

https://dre.pt/application/conteudo/178527. Acesso em: 16 nov. 2019.

Revisão gramatical realizada por: Maurício José Morais Costa

E-mail: mauricio.jmc@outlook.com 